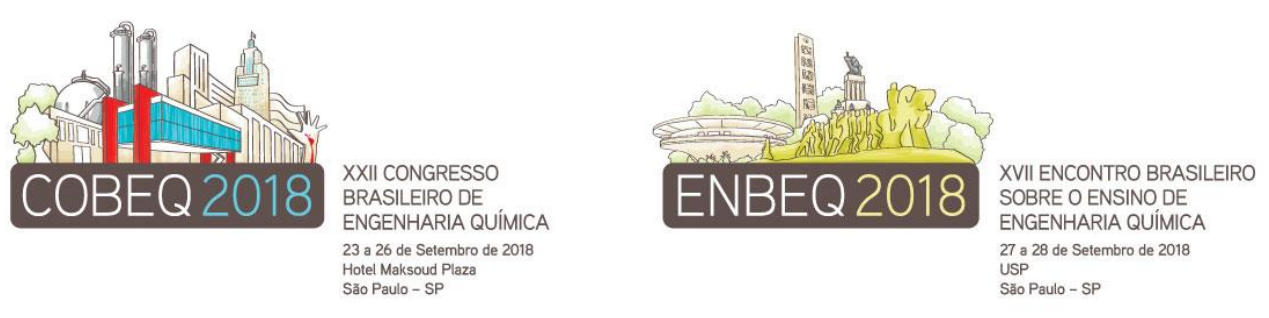

\title{
OBTENÇÃO DE TRIGLICERÍDEOS DIETÉTICOS DO TIPO MLM A PARTIR DA ACIDÓLISE ENZIMÁTICA DE ÓLEO DE ALGODÃO
}

\author{
BASSAN ${ }^{1}$, OLIVEIRA CG ${ }^{2}$, HATANAKA RR ${ }^{3}$, MONTI $^{1}$ e PAULA AV ${ }^{2}$ \\ ${ }^{1}$ Universidade Estadual Paulista (UNESP), Faculdade de Ciências Farmacêuticas, Campus \\ Araraquara, Departamento Alimentos e Nutrição \\ ${ }^{2}$ Universidade Estadual Paulista (UNESP), Faculdade de Ciências Farmacêuticas, Campus \\ Araraquara, Departamento de Bioprocessos e Biotecnologia \\ ${ }^{3}$ Universidade Estadual Paulista (UNESP), Instituto de Química, Campus Araraquara, \\ CEMPEQC \\ E-mail para contato: ariela@fcfar.unesp.br
}

\begin{abstract}
RESUMO - o objetivo do presente trabalho foi otimizar a síntese enzimática de triglicerídeos dietéticos do tipo MLM, por acidólise enzimática do óleo de algodão $e$ ácidos graxos de cadeia média em reator de tanque agitado. O produto gerado partir da acidólise foi analisado em cromatográfo gasoso para posterior cálculo de grau de incorporação e análise da posição sn-2. O melhor resultado de grau de incorporação (36,63 $\pm 0,23 \%)$ foi obtido utilizando-se a lipase Lipozyme RM IM e ácido cáprico. Com relação à posição sn-2 a composição em ácidos graxos da mesma foi mantida intacta uma vez que se utilizou uma lipase 1,3 específica.
\end{abstract}

\section{INTRODUÇÃO}

Os triglicerídeos do tipo MLM são aqueles que possuem ácidos graxos de cadeia média (M), nas posições $s n-1$ e $s n-3$, e de cadeia longa (L), na posição $s n-2$ da molécula de triacilglicerídeo. $\mathrm{O}$ interesse por este tipo de triglicerídeos aumentou, uma vez que apresentam baixo valor calórico e cuja ingestão é indicada para pessoas com deficiências metabólicas (Nunes et al., 2011 a, b; Caballero et al., 2014).

O objetivo do presente trabalho foi otimizar a síntese enzimática de triglicerídeos dietéticos do tipo MLM, por acidólise do óleo de algodão, e ácidos graxos de cadeia média em reator de tanque agitado. Para as reações de acidólise utilizou-se óleo de algodão devido ao alto valor nutricional que apresenta, além de ter sido pouco explorado neste tipo de reação visando-se à obtenção de TAGs dietéticos.

\section{MATERIAL E MÉTODOS}

\subsection{Reações de Acidólise}




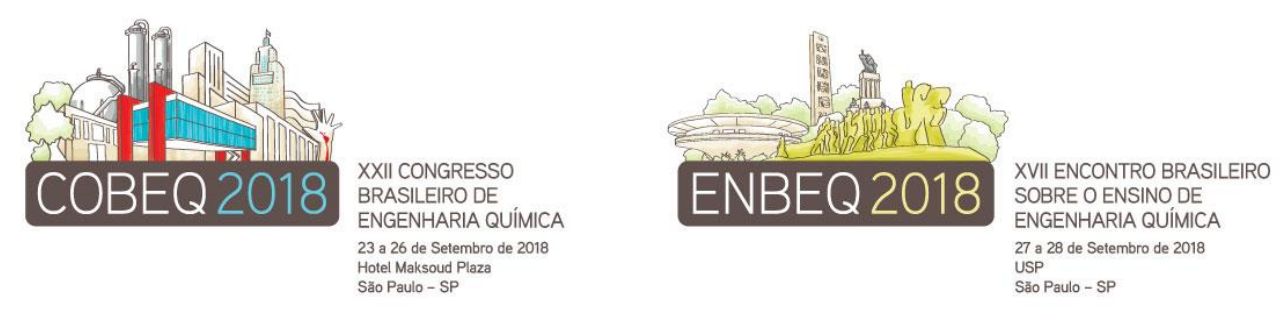

Reações de acidólise para síntese de triglicerídeos do tipo MLM: as reações de acidólise foram empregaram o óleo de algodão e os ácidos graxos (caprílico e/ou cáprico), em reator de vidro encamisado (diâmetro interno de $3 \mathrm{~cm}$, altura de $6 \mathrm{~cm}$ ), operando em regime batelada. Foram avaliadas três lipases comerciais imobilizadas (Lipozyme TL IM, Lipozyme RM IM e Novozym 435). As reações (30 g de meio reacional) ocorreram sob temperatura de $45^{\circ} \mathrm{C}$, com agitação constante de $400 \mathrm{rpm}$ em agitador magnético, razão molar óleo:ácido (1:2) e porcentagem de biocatalisador de $8 \%$.

\subsection{Análises do Produto}

Purificação e metilação dos triglicerídeos modificados: o excesso de ácidos graxos livres do produto foi neutralizado utilizando uma solução de hidróxido de potássio hidroalcoólica, como o descrito por Wang et al. (2012). Após a neutralização, o produto foi metilado para geração de ésteres metílicos (método ISO 12966-2, 2011, modificado).

Composição em ácidos graxos: a composição em ácidos graxos foi determinada por cromatografia gasosa, de acordo com o método Ce 2-66 da American Oil Chemist's Society (2011). O grau de incorporação foi calculado partir da Equação 1 (Casas-Godoy et al., 2013).

$$
G I(\%)=\frac{M F A}{M T} * 100
$$

Onde: MFA é o número de mols de ácidos graxos de cadeia média (C8:0 ou C10:0) no triglicerídeo e MT é o número de mols totais de ácidos graxos no triglicerídeo.

Análise da posição $S n$-2: a composição em ácidos graxos na posição 2 do triglicerídeo foi analisada de acordo com Caballero et al. (2014, modificado).

\section{RESULTADOS E DISCUSSÃO}

Na Tabela 1 estão apresentados os valores do grau de incorporação dos ácidos graxos cáprico e caprílico no óleo de algodão, utilizando as diferentes lipases comerciais (Lipozyme RM IM, Lipozyme TL IM e Novozym 435).

O GI variou de $21,80 \pm 3,12$ a 36,63 $\pm 0,23 \%$ sendo que a Lipozyme RM IM forneceu melhor desempenho de incorporação (Tabela 1). As maiores incorporações foram obtidas com ácido cáprico ( $\mathrm{C} 10: 0)$, pelo fato deste possuir uma cadeia carbônica mais longa, em comparação ao ácido caprílico (C8:0). A literatura relata que a especificidade destas lipases está diretamente relacionada ao tamanho da cadeia dos ácidos graxos, sendo que estas apresentam preferência por ácidos graxos de maior cadeia carbônica (Caballero et al., 2014; Rodrigues e Fernandez-Lafuente, 2010 a,b).

Para a determinação da posição $s n-2$ foi selecionado o melhor sistema enzima/ácido graxo, ou seja está análise foi realizada na reação utilizando ácido o cáprico e a Lipozyme RM IM. Na Tabela 2 estão apresentados o resultados obtidos a partir da determinação $s n-2$ do óleo de algodão sem modificação e modificado. 

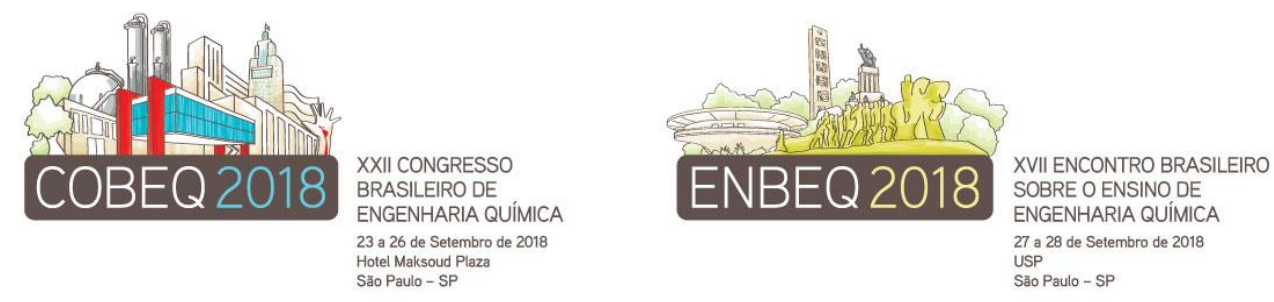

Tabela 1 - Grau de incorporação dos ácidos graxos cáprico e caprílico utilizando as diferentes lipases comerciais (Lipozyme RM IM, Lipozyme TL IM e Novozym 435

\begin{tabular}{ccc}
\hline & \multicolumn{2}{c}{ GI (\%) } \\
\hline & C10:0 & C8:0 \\
\hline Lipozyme RM IM & $36,63 \pm 0,23$ & $26,49 \pm 2,17$ \\
Lipozyme TL IM & $24,73 \pm 2,01$ & $22,92 \pm 4,35$ \\
Novozym 435 & $28,83 \pm 2,63$ & $21,80 \pm 3,12$ \\
*média \pm desvio padrão
\end{tabular}

Tabela 2 - Perfil composicional da posição $s n$ - 2 do óleo de algodão e do produto de reação utilizando C10:0 e Lipozyme RM IM

\begin{tabular}{ccc}
\hline & Óleo de algodão & $\begin{array}{c}\text { Óleo de algodão } \\
\text { modificado }\end{array}$ \\
\hline Ácidos Graxos & \% & $\%$ \\
\hline C10:0 & - & 3,58 \\
C16:0 & 2,87 & 2,35 \\
C18:0 & 0,89 & 0,92 \\
C18:1n9 & 32,24 & 31,54 \\
C18:3n3 & 60,78 & 57,43 \\
NI* & 3,32 & 4,14 \\
Total & - & 0,03 \\
\hline
\end{tabular}

*NI = não identificados

É possível observar (Tabela 2) que grande parte dos ácidos graxos insaturados de cadeia longa presentes na matéria prima ocupam a posição $s n-2$ do triglicerídeo. Após a acidólise, o perfil composicional desta posição foi praticamente inalterado. A posição $s n-2$ ser mantida já era um resultado espero, uma vez que se empregou uma lipase $s n-1,3$ regioseletiva (Mustafa et al., 2016).

Sabe-se que esta conformação (MLM) de triglicerídeo garante uma melhor absorção de ácidos graxos de cadeia longa (oleico e linoleico), já que a ingestão dos mesmos está associada à prevenção de doenças como, por exemplo, as doenças cardíacas, doenças relacionadas à má absorção lipídica, doenças inflamatórias, câncer, entre outras (Moreira et al., 2017 e Wang et al., 2012).

\section{CONCLUSÃO}

Os melhores resultados de grau de incorporação para o óleo de algodão $(36,63 \pm 0,23$ $\%$ ) foram obtidos utilizando-se ácido cáprico (C10:0) e a enzima Lipozyme RM IM. Além de um GI satisfatório, após a acidólise enzimática, a posição sn-2 manteve-se praticamente intacta. 

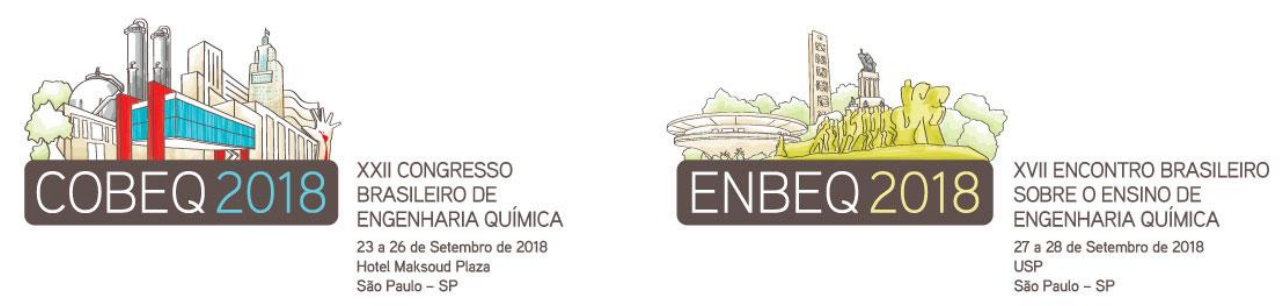

\section{AGRADECIMENTOS}

Fundação de Amparo à Pesquisa do Estado de São Paulo, FAPESP (Processo 2017/11482-7), CNPq (Processo 446371/2014-9), Unesp (PADC 2014/14-I).

\section{REFERÊNCIAS}

American Oil Chemists' Society. Official Methods and Recommended Practices of the AOCS, 2011 6th ed. AOCS Press.

CABllero E, SOTO C, Olivares A, Altamirano C, Potential use of avocado oil on structured Lipids MLM type production catalysed by commercial immobilised lípases. Plos one, v.9, p.1-7, 2014.

CASAS-GODOY L, MARTY A, SANDOVAL G, FERREIRA-DIAS S, Optimization of medium chain length fatty acid incorporation into olive oil catalyzed by immobilized Lip2 from Yarrowia lipolytica. Biochemical Engineering Journal, v.77, p.20 - 27, 2013.

ISO 12966-2 (2011). Animal and vegetable fats and oils - Gas chromatography of fatty acid methyl esters - Part 2: Preparation of methyl esters of fatty acids. International Organization for Standardization, Switzerland.

MOREIRA DKT, RACT JNR, RIBEIRO APB, MACEDO GA, Production and characterization of structured lipids with antiobesity potential and as a source of essential fatty acids. Food Research International, 2017; //dx.doi.org/10.1016/j.foodres.2017.06.034.

MUSTAFA A, KARMALI A, ABDELMOEZ W, Optimisation and economic assessment of lipasecatalysed production of monoesters using Rhizomucor miehei lipase in a solvent-free system. Journal of Cleaner Production, v.137, p.953-964, 2016.

NUNES PA, PIRES-CABRAL P, FERREIRA-DIAS S. Production of olive oil enriched with medium chain fatty acids catalysed by commercial immobilised lipases, Food Chemistry, v.127, p.993-998, 2011 a.

NUNES PA, PIRES-CABRAL P, GUILLEN M, VALERO F, LUNA D, FERREIRA-DIAS, S, Production of MLM-type structured lipids catalyzed by immobilized heterologous Rhizopus oryzae lipase. Journal American Oil Chemistry Society, v.88, p.473-480, 2011 b.

RODRIGUES RC, FERNANDEZ-LAFUENTE R, Lipase from Rhizomucor miehei as an industrial biocatalyst in chemical process. Journal of Molecular Catalysis B: Enzymatic, v.64, p.1-22, 2010.

RODRIGUES RC, FERNANDEZ-LAFUENTE R, Lipase from Rhizomucor miehei as a biocatalyst in fats and oils modification. Journal of Molecular Catalysis B: Enzymatic, v.66, p.15-32, 2010.

WANG Y, XIA L, XU X, XIE L, DUAN Z, Lipase-catalyzed acidolysis of canola oil with caprylic acid to produce medium-, long- and medium-chain-type structured lipids. Food and Bioproducts Processing, v.4, p.707-712, 2012. 